\title{
PROCESSOS OXIDATIVOS NA FRAÇÃO LIPÍDICA DE ALIMENTOS
}

\author{
DENISE JOSINO SOARES* \\ TARLIANE MARTINS TAVARES** \\ ISABELLA MONTENEGRO BRASIL*** \\ RAIMUNDO WILANE DE FIGUEIREDO**** \\ PAULO HENRIQUE MACHADO DE SOUSA*****
}

\begin{abstract}
A presente revisão de literatura teve como objetivo explicar os mecanismos da oxidação lipídica, mostrando os principais métodos de detecção da sua ocorrência em alimentos. Foram abordados ainda o seu efeito nos alimentos e em que etapa do processamento a oxidação costuma ocorrer. Diversas metodologias foram criadas com o intuito de quantificar e identificar a ocorrência da oxidação de lipídios em alimentos, algumas envolvem métodos químicos e físico-químicos e outras são realizadas por meio de testes sensoriais. A utilização de métodos de quantificação juntamente com a aplicação das avaliações sensoriais torna-se útil para analisar como o aumento da ocorrência de processos oxidativos irá ser detectada, representando alternativa para se estimar a manutenção da qualidade sensorial e a aceitação do produto no mercado consumidor
\end{abstract}

PALAVRAS-CHAVE: RANCIDEZ OXIDATIVA; AUTO-OXIDAÇÃO DE LIPÍDIOS; FOTO-OXIDAÇÃO.

* Doutoranda em Ciência e Tecnologia de Alimentos, Universidade Federal do Ceará (UFC), Fortaleza, CE (e-mail: denisejosino@hotmail.com).

** Doutoranda em Agronomia, UFC, Fortaleza, CE (e-mail: agrotmt@yahoo.com.br).

*** Doutora em Bioquímica, Professora, Departamento de Tecnologia de Alimentos, UFC, Fortaleza, CE (e-mail: isabella@ufc.br).

**** Doutor em Ciência dos Alimentos, Professor, Departamento de Tecnologia de Alimentos, UFC, Fortaleza, CE (e-mail: figueira@ufc.br).

***** Doutor em Ciência e Tecnologia de Alimentos, Professor, Instituto de Cultura e Arte, UFC, Fortaleza, CE (e-mail: phenriquemachado@gmail.com). 


\section{INTRODUÇÃO}

Os lipídios (óleos e gorduras) constituem os principais componentes insolúveis em água presentes em alimentos, sendo encontrados quase sempre na forma de triglicerois (ARAÚJO, 2008). Compostos por ácidos graxos, os triglicerois desempenham importante papel na qualidade sensorial dos alimentos e em seu valor nutritivo por serem fontes de energia, de ácidos graxos essenciais (principalmente o ácido linoleico) e de vitaminas lipossolúveis (MASUCHI et al., 2008).

Em alimentos, os ácidos graxos podem ser saturados ou insaturados. Os ácidos graxos insaturados apresentam as configurações cis ou trans (ARAÚJO, 2008) e são as estruturas mais susceptíveis ao processo oxidativo (RAMALHO e JORGE, 2006). Os ácidos graxos na configuração trans estão presentes naturalmente em gorduras de animais ruminantes (devido ao processo de biohidrogenação) e em produtos alimentícios industrializados, como margarinas, cremes vegetais, biscoitos, sorvetes e outros (devido ao processo de hidrogenação de óleos vegetais). Sua ingestão excessiva representa fator de risco para a ocorrência de doença arterial coronariana (CHIARA, SICHIERI e CARVALHO, 2003). Assim, pode-se afirmar que os lipídios da dieta desempenham importante papel no risco de desenvolvimento de diversas doenças crônicas (BERTOLINO et al., 2006).

Durante o processamento e o armazenamento de alimentos que contém lipídios em sua constituição, o principal mecanismo de deterioração é a oxidação lipídica que pode tornar o alimento inaceitável sensorialmente, bem como produzir substâncias potencialmente tóxicas (TABEE et al., 2008).

Devido aos problemas ocasionados pela oxidação lipídica é de suma importância a elucidação desse mecanismo em alimentos, bem como a aplicação dos métodos de quantificação e controle. Diante do exposto, o presente trabalho teve como objetivo mostrar os mecanismos que levam à oxidação lipídica, sua quantificação e suas formas de controle.

\section{OXIDAÇÃO DE LIPÍDIOS EM ALIMENTOS}

A deterioração de alimentos ocorre durante seu processamento e armazenamento, podendo estar relacionada com processos oxidativos (HINNEBURG, DORMAN e HILTUNEN, 2006) e microbiológicos que levam ao desenvolvimento de características que tornam o produto impróprio para o consumo.

Alimentos com elevado teor de lipídios são altamente perecíveis devido, principalmente, à ocorrência de processos oxidativos aos quais os óleos estão expostos (SILVA, ASCHERI e SOUZA, 2010).

A oxidação dos constituintes lipídicos constitui reação importante que limita a vida-deprateleira de vários alimentos. As alterações na qualidade dos alimentos podem ser percebidas pelas mudanças nas características sensoriais, no valor nutricional e pela produção de compostos potencialmente tóxicos (MARIUTTI e BRAGAGNOLO, 2009). Esses processos de oxidação lipídica envolvem a reação de ácidos graxos insaturados com o oxigênio. Dessa forma, a estabilidade de determinado alimento fonte de lipídios depende da proporção de ácidos graxos saturados e insaturados (TIRITAN e BEUX, 2006).

Os processos oxidativos abrangem a oxidação enzimática, a fotoxidação e a autoxidação. A oxidação por via enzimática ocorre pela ação das lipoxigenases (linoleato: oxigênio oxido-redutase - EC 1.13.11.12) que são dioxigenases que catalisam a adição do oxigênio molecular ao sistema cis, cis, 1,4 - pentadieno dos ácidos graxos poliinsaturados, formando hidroperóxidos (SILVA et al., 2001). A lipoxigenase gera aromas voláteis similares aos produzidos durante a auto-oxidação, embora as proporções relativas dos produtos possam variar muito, dependendo da especificidade da enzima e das condições da reação.

A fotoxidação ocorre pela radiação ultravioleta na presença de fotossensibilizadores (como 
a clorofila, mioglobina e riboflavina, dentre outros), que promovem a transformação de oxigênio triplete $\left(3 \mathrm{O}_{2}\right)$ em oxigênio singlete $\left(\mathrm{O}_{2}\right)$, sua forma mais reativa, cujas degradações posteriores originam aldeídos, álcoois e hidrocarbonetos. A autoxidação representa o principal mecanismo de oxidação de lipídios em alimentos, ocorrendo em três etapas (iniciação, propagação e terminação) que levam à formação de radicais livres (RAMALHO e JORGE, 2006).

Segundo Fogaça e Sant'ana (2009), os radicais livres são espécies químicas que apresentam um ou mais elétrons desemparelhados no seu último orbital, o que os tornam altamente reativos. Devido à essa característica de alta reatividade, tais espécies químicas podem alterar a estrutura e a função de componentes celulares e extracelulares, contribuindo para o surgimento de doenças (BLOMHOFFI et al., 2006).

Os principais problemas decorrentes da oxidação lipídica são as alterações sensoriais, envolvendo o desenvolvimento de aromas desagradáveis (ranço) (DEGÁSPARI e WASZCZYNSKYJ, 2004), o que irá tornar o alimento impróprio para consumo. A rancidez oxidativa ocorre em lipídios contendo ácidos graxos insaturados, que podem sofrer oxidação formando aldeídos, cetonas, ácidos, álcoois e hidrocarbonetos. Esses identificam o fenômeno da rancidez (QUAST e AQUINO, 2004) (FELLOWS, 2006), que representa importante causa de rejeição de produtos pelo consumidor, podendo provocar alterações na sua qualidade nutricional, devido à degradação de vitaminas lipossolúveis e de ácidos graxos essenciais (RAMALHO e JORGE, 2006), principalmente do ácido linoleico (rapidamente perdido).

A rancidez hidrolítica e a rancidez oxidativa (autoxidação) representam grandes problemas técnicos para a indústria de alimentos (OSAWA, GONÇALVES e RAGAZZI, 2006).

A magnitude de processos oxidativos depende de fatores do próprio alimento (teor de água e a presença de substância pró-oxidantes) e das condições de processamento e armazenamento, como sua exposição a atmosferas gasosas e à luz (TABEE et al., 2008). Segundo Waraho, McClements e Decker (2011), alguns componentes presentes no alimento, como ácidos graxos livres e fosfolipídios podem aumentar potencialmente as taxas de oxidação lipídica. Por outro lado, as proteínas são capazes de inibir a oxidação lipídica.

Segundo Degáspari e Waszczynskyj (2004), os processos oxidativos ocorrem principalmente quando o alimento apresenta valores de atividade de água inferiores a 0,3 , pois nessa faixa atinge-se a zona de adsorção que propicia a ação catalítica dos metais e que irá favorecer o desenvolvimento das reações de oxidação de lipídios.

Emulsões de óleo em água, observadas em alimentos como leite, maionese, molhos para saladas, sopas, bebidas e cremes, podem sofrer oxidação lipídica rapidamente devido à sua grande área superficial (WARAHO, McCLEMENTS e DECKER, 2011).

Durante o processamento de alimentos por meio de fritura, o óleo utilizado também é passível de sofrer diversas reações, incluindo os processos oxidativos (hidrólise e oxidação) e a polimerização (TABEE et al., 2008).

Ping-Delfos e Soares (2011), estudando a influência do cálcio e da vitamina D nos processos oxidativos da fração lipídica de diferentes refeições, observaram que esses elementos aumentam de forma aguda a incidência de oxidação lipídica.

Alguns corantes artificiais, como a eritrosina B e floxina B, que são amplamente utilizados em produtos de confeitaria e bebidas atuam acelerando a oxidação do ácido linoleico, ou seja, apresentam ação pró-oxidante (PAN, USHIO e OHSHIMA, 2005).

\section{MÉTODOS DE AVALIAÇÃO DO ESTADO OXIDATIVO DOS LIPÍDIOS EM ALIMENTOS}

Os processos oxidativos de lipídios representam o principal parâmetro de controle físico-químico para definir o prazo de validade de alguns alimentos processados (DEGÁSPARI e 
WASZCZYNSKYJ, 2004), tornando importante o conhecimento de técnicas para a sua detecção nos alimentos.

Existem diferentes formas de se avaliar o estado oxidativo de óleos ou de frações lipídicas dos alimentos. Dentre os testes mais utilizados estão: índice de acidez da fração lipídica (rancidez hidrolítica), índice de peróxido, índice de anisidina (rancidez oxidativa) (LIMA e SOUSA, 2001) e determinação de dienos conjugados, substâncias reativas ao ácido tiobarbitúrico (FOGAÇA e SANT'ANA, 2009). Esses métodos geralmente envolvem a extração da fração lipídica do alimento em estudo seguido de análise.

A medida do índice de acidez da fração lipídica corresponde ao número de $\mathrm{mg}$ de $\mathrm{KOH}$ necessário para neutralizar os ácidos graxos livres em $1 \mathrm{~g}$ de amostra (CECCHI, 2003). O valor do índice aumenta com a incidência de processos oxidativos porque durante a oxidação maior quantidade de ácidos graxos livres é formada, não sendo possível ultrapassar o valor de 2,0\% em ácido oleico (OSAWA, GONÇALVES e RAGAZZI, 2006). Essa medida evidencia o estado de conservação da fração lipídica do alimento, pois avalia a quantidade de ácidos graxos provenientes dos processos oxidativos. Trata-se de variável que relaciona os aspectos de qualidade da matéria-prima, do processamento e do armazenamento do alimento (TIRITAN e BEUX, 2006). O procedimento baseia-se na dissolução do lipídio em solvente, previamente neutralizado, seguido de titulação com a solução padrão de $\mathrm{NaOH}$, utilizando-se fenolftaleína como indicador (AOCS, 2004). Essa metodologia pode ser realizada em potenciômetro, sem a utilização do indicador de fenolftaleína, sendo o ponto final medido quando a solução atinge pH 8,8 (OSAWA, GONÇALVES e RAGAZZI, 2006).

A medida do índice de peróxido determina as substâncias em miliequivalentes de peróxido por $1000 \mathrm{~g}$ de amostra. É realizada pela reação de iodeto de potássio com efeito oxidante dos peróxidos. O iodo liberado pela reação é titulado com tiossulfato de sódio (TIRITAN e BEUX, 2006).

Define-se o valor anisidina como 100 vezes a densidade óptica de uma solução contendo $1,0 \mathrm{~g}$ de gordura em cada $100 \mathrm{~mL}$ da mistura de solventes e reagentes, medido em comprimento de onda de $350 \mathrm{~nm}$ em célula de $1 \mathrm{~cm}$ usando-se espectrofotômetro (AOCS, 2004). O conteúdo de peróxido nos lipídeos oxidados de origem animal ou vegetal são componentes transitórios que se decompõem em diversos compostos carbonílicos e outros. Portanto, o reagente de $p$-anisidina forma o produto da condensação com aldeídos (2-alcenais e 2,4-alcadienais) sob condições ácidas (OSAWA, GONÇALVES e RAGAZZI, 2008). Segundo Osawa (2005), o método sofre interferência da umidade, pois a reação $p$-anisidina e os aldeídos resultam na formação de água.

Segundo Lima e Abdalla (2001), os dienos conjugados são formados durante a oxidação de ácidos graxos poliinsaturados (principalmente linoleico e linolênico) e podem ser detectados pela sua absorção no ultravioleta, apresentando absorção máxima entre 230 e $235 \mathrm{~nm}$. A análise de quantificação de dienos conjugados pode ser realizada segundo metodologia proposta pela AOCS (2004).

A quantificação do malonaldeído (MDA), produto tóxico que surge com a degradação dos ácidos graxos, é utilizada como importante marcador na avaliação do dano oxidativo em alimentos (DOTAN, LICHTENBERG e PINCHUK, 2004).

O teste de ácido tiobarbitúrico (TBA), medida da rancidez, baseia-se na reação do ácido-2tiobarbitúrico com os aldeídos formados durante a oxidação de lipídios (MDA) (JORGE e JANIERE, 2005) e pode ser efetuado segundo metodologia proposta pelo Instituto Adolfo Lutz (2008). Essa medida pode ser realizada por absorção no visível $(532 \mathrm{~nm})$ ou por fluorescência ( $\lambda$ exc $=515 \mathrm{~nm}$ e $\lambda e m=553 \mathrm{~nm})$ (LIMA e ABDALLA, 2001). A quantificação dos malonaldeídos fornece o índice indireto de injúria oxidativa resultante de peroxidação lipídica (SOUZA JR., OLIVEIRA e PEREIRA, 2005).

Nova metodologia para determinação da oxidação do colesterol em alimentos foi proposta por Raith et al. (2005), que permite obter resultados rápidos e precisos usando cromatografia e espectrofotômetria de massa. 
Pode-se avaliar o estado oxidativo de alimentos lipídicos mediante teste sensoriais. A avaliação sensorial permite intervir ao longo do tempo no aparecimento dos produtos de degradação dos lipídios, que causam sabores e odores estranhos ao alimento e que são facilmente detectados sensorialmente. É considerada determinação autêntica e de importância, pois mede e representa o que o consumidor percebe mediante testes de aceitabilidade (SILVA, BORGES e FERREIRA, 1999).

\section{MECANISMOS DE PROTEÇÃO DE ALIMENTOS CONTRA A OCORRÊNCIA DE PROCESSOS OXIDATIVOS}

A preservação das características originais dos alimentos durante o maior tempo possível continua sendo grande desafio para a indústria de alimentos (LIMA e BRUNO, 2007). Assim, a prevenção da ocorrência de processos oxidativos durante o processamento e o armazenamento de alimentos assume importância fundamental para manter a segurança e a qualidade desses produtos (REY et al., 2005).

A indústria de alimentos busca sempre melhorar o conteúdo nutricional de seus produtos e para isto precisa aprimorar aspectos de qualidade como a prevenção da rancidez, devido à presença de maior quantidade de ácidos graxos poliinsaturados na composição dos alimentos (WARAHO, McCLEMENTS e DECKER, 2011).

Sabe-se que a oxidação de lipídios não pode ser paralisada e que quando iniciada ocorre em alta velocidade, porém alguns cuidados podem ser tornados com a finalidade de minimizar os processos oxidativos em alimentos (MASUCHI et al., 2008). Dessa forma, diferentes componentes são adicionados aos alimentos a fim de reduzir mudanças durante o seu processamento, armazenamento e comercialização.

Evitar a ocorrência de processos oxidativos em alimentos exige a redução da ação dos fatores que os favoreçam. Assim, deve-se preservar o alimento dos fatores (temperatura e luz) que beneficiam o processo de composição dos radicais livres, impedindo a presença de traços de metais e o contato com o oxigênio (RAMALHO e JORGE, 2006).

Segundo Soto-Rodríguez et al. (2008) é necessário maior desenvolvimento das técnicas de processamento e de armazenamento de produtos alimentícios com a finalidade de minimizar a ocorrência da oxidação lipídica.

Em carnes processadas, a adição de nitritos representa alternativa para minimizar a ocorrência dos processos oxidativos e o surgimento de sabores e odores desagradáveis nesses produtos (REY et al., 2005).

Os antioxidantes são substâncias que inibem a oxidação lipídica, ajudando a prevenção da deterioração de alimentos lipídicos (MASUCHI et al., 2008). Esses compostos atuam estabilizando ácidos graxos mediante reação com radicais livres, quelando íons metálicos e interrompendo a fase de propagação da oxidação lipídica (DEGÁSPARI e WASZCZYNSKYJ, 2004).

Compostos antioxidantes podem ser adicionados com a finalidade de inibir ou retardar a oxidação dos componentes lipídicos dos alimentos. Os mais utilizados são os sintéticos hidroxianisolbutilado (BHA), hidroxitolueno butilado (BHT), butilhidroquinona terciário (TBHQ) e os naturais (tocoferois, ácidos fenólicos e extratos de alecrim e sálvia) (RAMALHO e JORGE, 2006). Devido a preocupação e busca do consumidor por alimentos mais saudáveis, as indústrias têm utilizado, preferencialmente, antioxidantes naturais (WARAHO et al., 2011).

Os antioxidantes podem ser adicionados diretamente ao alimento ou usados como filme de cobertura. Abreu et al. (2011) estudaram o armazenamento de peixes utilizando filmes de cobertura com antioxidantes. Observaram que os peixes cobertos com esses filmes apresentaram menores valores de ácidos graxos livres, malonaldeido e peróxidos do que os que não estavam recobertos.

Os pecuaristas têm adicionado substâncias derivadas de plantas, tais como ervas secas e óleos essenciais, na alimentação de animais para reduzir a oxidação lipídica em produtos cárneos. 
Tais substâncias são fontes de antioxidantes naturais como os carotenoides e flavonoides (KARAMI et al., 2011).

Lee, Chien e Chen (2008), estudando a eficiência de antioxidantes sintéticos e naturais na redução da oxidação de ovos e de carne de porco fritos verificaram que o Trolox, o $\mathrm{BHA}$ e as vitaminas $\mathrm{C}$ e $\mathrm{E}$ são eficientes no retardamento dos processos oxidativos desses produtos.

REY et al. (2005), avaliando a aplicação de antioxidantes naturais obtidos de amora silvestre, da beterraba e da erva do salgueiro em carne de porco cozida, observaram que essas substâncias são potentes agentes de proteção à ocorrência da oxidação desse produto.

Silva, Ascheri e Souza (2010) estudaram o efeito de compostos com atividade antioxidante naturalmente presentes nos vegetais. Constataram que o tocoferol contido no óleo de vegetais exerce importante papel na sua estabilidade oxidativa.

Substâncias, como o ácido carnósico e a curcumina apresentam efeito na redução da oxidação em alimentos. Zunin et al. (2010), estudando o efeito do ácido carnósico na proteção da oxidação de lipídios em óleo de oliva virgem, verificaram que a elevação da concentração desse ácido provoca aumento da proteção dos processos oxidativos. A utilização de curcumina para reduzir a produção de malonaldeídos em produtos cárneos foi relatada por Karami et al. (2011) que constataram a eficiência dessa substância natural no controle de processos oxidativos de lipídios em alimentos.

\section{EXEMPLOS DE OXIDAÇÃO DE LIPÍDIOS EM ALIMENTOS}

Diversos estudos têm sido realizados com o intuito de verificar a ocorrência de oxidação lipídica em alimentos e de como minimizar os processos oxidativos. Machado e Druzian (2009) ao estudarem a estabilidade oxidativa da manteiga de garrafa, produzida artesanalmente, constataram que as amostras já se apresentavam com certo grau de oxidação no período inicial das análises e que esses valores se mantiveram constantes durante 7 meses de armazenamento. Isso indica que as manteigas passaram por processos oxidativos no momento de sua produção e que 0 armazenamento desse tipo de produto a $5^{\circ} \mathrm{C}$ por até 7 meses controla o avanço das reações de oxidação.

Descalzo et al. (2008), avaliando a estabilidade de carne de búfalo durante 25 dias de armazenamento a $-20^{\circ} \mathrm{C}$, verificaram aumento na produção de malonaldeído durante esse período, indicando que mesmo em temperaturas baixas podem ocorrer processos oxidativos na carne estudada.

Cella, Regitano-D'arce e Spoto (2002), analisando a aceitabilidade sensorial do óleo de soja utilizado em frituras, observaram que os julgadores foram unânimes em perceber os atributos "leve", "suave" e "característico de fritura" e "sem cheiro ou gosto de óleo de fritura" no alimento. O ranço esperado não apareceu em nenhum dos seis períodos de horas acumuladas das frituras durante o experimento, os quais não diferiram significativamente do primeiro período.

Ambrósio, Guerrra e Mancini Filho (2001) estudaram sensorialmente duas marcas de manteiga de garrafa. Observaram diferenças estatísticas, ao nível de significância de $5 \%$, quanto aos atributos odor característico, cor (translucidez, tonalidade), textura não oral e oral, sabor metálico e característico. Afirmaram que as características sensoriais inerentes a cada produto desempenham papel relevante em sua aceitabilidade. A cor, o odor, e o sabor são características importantes que representam o padrão de qualidade dos alimentos, visto que a oxidação lipídica causa alterações sensoriais peculiares.

Römer, Majchzak e Elmadfa (2008) avaliaram três tipos diferentes de massa, sendo uma preparada sem lipídios, outra com óleo de colza fresco e a última com óleo de colza oxidado. Observaram aumento no índice de peróxido nas massas preparadas com óleo, principalmente quando foi utilizado óleo de colza oxidado. Tais autores avaliaram sensorialmente as diferentes massas produzidas quanto aos atributos aparência, odor, sabor e textura. Constataram que a 
massa preparada com óleo de colza oxidado apresentou maiores índices de rejeição por parte dos julgadores.

Soares et al. (2012a) avaliaram a estabilidade sensorial de amêndoas de castanha de caju, armazenadas em embalagem laminada à temperatura ambiente (aproximadamente $\left.25^{\circ} \mathrm{C}\right)$, durante 6 meses. Os referidos autores não verificaram diferença significativa $(p>0,05)$ quanto aos atributos cor, sabor e crocância durante todo o período de armazenamento. Em outro trabalho, Soares et al. (2012b) quantificaram o índice de acidez da fração lipídica das amêndoas armazenadas nas mesmas condições descritas anteriormente. Obtiveram resultados semelhantes durante os 6 meses de armazenamento, evidenciando que as amêndoas não sofreram processos de rancificação hidrolítica, o que contribuiu para a boa aceitação das amêndoas durante esse período.

Trindade et al. (2010) estudaram a estabilidade oxidativa de mortadela contendo óleo de soja, armazenada a $0^{\circ} \mathrm{C}$ durante 60 dias. Verificaram mediante aceitação sensorial que a substituição do toucinho suíno pelo óleo de soja não afetou $(p>0,05)$ a estabilidade das mortadelas durante 46 dias de armazenamento. Constataram que a utilização de óleo de soja na elaboração de mortadelas, substituindo 50 a $100 \%$ do toucinho suíno, não prejudicou a estabilidade oxidativa e sensorial desses produtos, durante a estocagem.

\section{CONCLUSÃO}

Os processos oxidativos que ocorrem nos lipídios representam uma das principais causas da redução da vida-de-prateleira dos produtos alimentícios. A elucidação dos mecanismos desses processos, bem como suas formas de controle são de elevada importância para a indústria alimentícia.

Existem diferentes formas de proteger o alimento contra a ocorrência de processos oxidativos, cabendo ao produtor selecionar a tecnologia de preservação que melhor se adapte à natureza química do produto.

Diversas metodologias foram criadas com o intuito de quantificar e identificar a ocorrência da oxidação de lipídios em alimentos, algumas envolvem métodos químicos e físico-químicos e outras são realizadas por meio de testes sensoriais. A utilização de métodos de quantificação juntamente com a aplicação das avaliações sensoriais torna-se útil para analisar como o aumento da ocorrência de processos oxidativos irá ser detectada, representando alternativa para se estimar a manutenção da qualidade sensorial e a aceitação do produto no mercado consumidor.

\section{ABSTRACT \\ OXIDATIVE PROCESSES IN LIPID FRACTION OF FOOD}

This literature review aims to explain the mechanisms of lipid oxidation, presenting the main methods of detecting its occurrence in foods. Were also addressed its effect on food and the steps in which the oxidation process usually occurs. Several methods were developed aiming at quantifying and identifying the occurrence of lipids oxidation on food, some of them including chemical and physicochemical methods while others were based on sensory analysis. The utilization of quantifying methods combined with sensory analysis can be very useful to analyze ways of detecting an increase on oxidative processes and, therefore it constitutes an alternative to estimate maintenance of food sensory qualities and the acceptance of the product.

KEY-WORDS: OXIDATIVE RANCIDITY; LIPID AUTO-OXIDATION; PHOTO-OXIDATION.

\section{REFERÊNCIAS}

1 ABReU, D. A. P.; LOSADA, P. P.; MAROTO, J.; CRUZ, J. M. Lipid damage during frozen storage of Atlantic halibut (Hippoglossus hippoglossus) in active packaging film containing antioxidants. Food Chemistry, v. 126, n. 1, p. 315-320, 2011. 
2 AOCS. American Oil Chemists' Society. Official methods and recommended practices of the American Oil Chemists Society. $5^{\text {th }}$ ed. Champaign, 2004.

3 AMBRÓSIO, C. L. B.; GUERRA, N. B.; MANCINI FILHO, J. Características de identidade, qualidade e estabilidade da manteiga de garrafa. Parte I. Características de identidade e qualidade. Ciência e Tecnologia de Alimentos, v. 21, n. 3, p. 314-320, 2001

4 ARAÚJO, J. M. A. Química de alimentos: teoria e prática. 4ª Ed. Viçosa: Editora UFV, 2008, 596 p.

5 BERTOLINO, C. N.; CASTRO, T. G.; SARTORELLI, D. S.; FERREIRA, S. R. G.; CARDOSO, M. A. Influência do consumo alimentar de ácidos graxos trans no perfil de lipídios séricos em nipo-brasileiros de Bauru, São Paulo, Brasil. Cadernos de Saúde Pública, Rio de Janeiro, v. 22, n. 2, p. 357-364, 2006.

6 BLOMHOFFI, R.; CARLSEN, M. H.; ANDERSEN, L. F.; JACOBS JR.; D. R. Health benefits of nuts: potential role of antioxidants. British Journal of Nutrition, v. 96, n. 2, p.52-60, 2006.

7 CECCHI, H. M. Fundamentos teóricos e práticos em análise de alimentos. 2. ed. Campinas: Unicamp, 2003. Cap. 9.

8 CELLA, R. C. F; REGITANO-D'ARCE, M. A. B.; SPOTO, M. H. F. Comportamento do óleo de soja refinado utilizado em fritura por imersão com alimentos de origem vegetal. Ciência e Tecnologia de Alimentos, v. 22, n. 2, p. 111-116, 2002.

9 CHIARA, V. L.; SICHIERI, R.; CARVALHO, T. S. F. Teores de ácidos graxos trans de alguns alimentos consumidos no Rio de Janeiro. Revista de Nutrição, Campinas, v. 16, n. 2, p. 227-233, 2003.

10 DEGÁSPARI, C. H.; WASZCZYNSKYJ, N. Propriedades antioxidantes de compostos fenólicos. Visão Acadêmica, v. 5, n. 1, p. 33-40, 2004

11 DESCALZO, A. M.; ROSSETTI, L.; SANCHO, A. M.; GARCÍA, P. T.; BIOLATTO, A.; CARDUZA, F.; GRIGIONI, G. M. Antioxidant consumption and development of oxidation during ageing of buffalo meat produced in Argentina. Meat Science, v. 79, n. 3, p. 528-588, 2008.

12 DOTAN, Y.; LICHTENBERG, D.; PINCHUK, I. Lipid peroxidation cannot be used as a universal criterion of oxidative stress. Progress in Lipid Research, v. 43, n. 3, p. 200-227, 2004.

13 FELLOWS, P. J. Tecnologia do processamento de alimentos: princípios e práticas. 2. ed. Porto Alegre: Editora Artmed, 2006. $602 \mathrm{p}$.

14 FOGAÇA, F. H. S; SANT'ANA, L. S. Oxidação lipídica em peixes: mecanismo de ação e prevenção. Archives of Veterinary Science, v. 14, n. 2, p. 117-127, 2009.

15 HINNEBURG, I.; DORMAN, H. J.; HILTUNEN R. Antioxidant activities of extracts from selected culinary herbs and spices. Food Chemistry, London, v. 97, n. 1, p. 122-129, 2006.

16 IAL. Instituto Adolfo Lutz. Métodos físico-químicos para análise de alimentos. 6. ed. São Paulo, 2008.1020 p.

17 JORGE, N.; JANIERI, C. Avaliação do óleo de soja submetido ao processo de fritura de alimentos diversos. Revista Ciência e Agrotecnologia, v. 29, n. 5, p. 1001-1007, 2005

18 KARAMI, M.; ALIMON, A. R.; SAZILI, A. Q.; GOH, Y. M.; IVAN, M. Effects of dietary antioxidants on the quality, fatty acid profile, and lipid oxidation of longissimus muscle in Kacang goat with aging time. Meat Science, v. 88, n. 1, p. 102-108, 2011.

19 LEE, H. W.; CHIEN, J. T.; CHEN, B. H. Inhibition of cholesterol oxidation in marinated foods as affected by antioxidants during heating. Food Chemistry, London, v. 108, n. 1, p. 234-244, 2008.

20 LIMA, E. S.; ABDALLA, D. S. P. Peroxidação lipídica: mecanismos e avaliações em amostras biológicas. Revista Brasileira de Ciências Famacêuticas, v. 37, n. 3, p. 293-303, 2001.

21 LIMA, J. R.; BRUNO, L. M. Estabilidade de pasta de amêndoa de castanha de caju. Ciência e Tecnologia de Alimentos, Campinas, v. 27, n. 4, p. 816-822, 2007.

22 LIMA, J. R.; SOUSA, M. M. M. Influência do tipo de óleo utilizado para fritura na estabilidade de amêndoas de castanha de caju. Boletim do CEPPA, Curitiba, v. 19, n. 1, p. 43-52, 2001

23 MACHADO, B. A. S.; DRUZIAN, J. I. Análise da estabilidade e da composição em ácidos graxos em manteiga de garrafa produzida artesanalmente. Revista do Instituto Adolfo Lutz, v. 68, n. 2, p. 201-208, 2009.

24 MARIUTTI, L. R. B.; BRAGAGNOLO, N. A oxidação lipídica em carne de frango e o impacto da adição de sálvia (Salvia officinalis, L.) e de alho (Allium sativum, L.) como antioxidantes naturais. Revista do Instituto Adolfo Lutz, v. 68, n. 1, p. $1-11,2009$.

25 MASUCHI, M. H.; CELEGHINI, M. S.; GONÇAVES, L. A. G.; GRIMALDI, R. Quantificação de TBHQ (terc butil hidroquinona) e avaliação da estabilidade oxidativa em óleos de girassol comerciais. Química Nova, v. 31, n. 5, p. 1053-1057, 2008. 
26 OSAWA, C. C.; GONÇALVES, L. A. G.; RAGAZZI, S. Evaluation of the quality of pet foods using fast techniques and official methods. Ciência e Tecnologia de Alimentos, v. 28, p. 223-230, 2008.

27 OSAWA, C. C.; GONÇALVES, L. A. G.; RAGAZZI, S. Titulação potenciométrica aplicada na determinação de ácidos graxos livres de óleos e gorduras comestíveis. Quimica Nova, v. 29, n. 3, p. 593-599, 2006.

28 OSAWA, C. C. Testes rápidos (Kits) para avaliação da qualidade de óleos, gorduras e produtos que o contenham e sua correlação com os métodos oficiais da AOCS. Revista Analytica, n. 16, p. 68-69, 2005.

29 PAN, X.; USHIO, H.; OHSHIMA, T. Effects of molecular configurations of food colorants on their efficacies as photosensitizers in lipid oxidation. Food Chemistry, London, v. 92, n. 1, p. 37-44, 2005.

30 PING-DELFOS, W. C. S.; SOARES, M. Diet induced thermogenesis, fat oxidation and food intake following sequential meals: influence of calcium and vitamin D. Clinical Nutrition, v.30, n.3, p. 376-383, 2011.

31 QUAST L. B.; AQUINO A. D. Oxidação dos lipídios em café arábica (Coffea arábica I.) e café robusta (Coffea canephora P.). Boletim do CEPPA, Curitiba, v.22, n.2, p. 325-336, 2004.

32 RAITH, K.; BRENNER, C.; FARWANAH, H.; MULLER, G.; EDER, K.; NEUBERT, R. H. H. A new LC/APCI-MS method for the determination of cholesterol oxidation products in food. Journal of Chromatography A, v. 1067, n. 1-2, p. 207-211, 2005.

33 RAMALHO, V. C.; JORGE, N. Antioxidantes utilizados em óleos, gorduras e alimentos gordurosos. Química Nova, v. 29, n. 4 , p. $755-760,2006$

34 REY, A. I.; HOPIA, A.; KIVIKAR, R.; KAHKONEN, M. Use of natural food/plant extracts: cloudberry (Rubus Chamaemorus), beetroot (Beta Vulgaris "Vulgaris") or willow herb (Epilobium angu SOUZA stifolium) to reduce lipid oxidation of cooked pork patties. Food Science and Technology, v. 38, n. 4, p. 363-370, 2005.

35 RÖMER, J.; MAJCHZAK, D.; ELMADFA, I. Interaction between amino acid degradation, sensory attributes and lipid peroxidation in wheat rolls. European Journal of Lipid Science and Technology, v. 110, n. 6, p. 554-562, 2008.

36 SILVA, F. A. M.; BORGES, M. F. M.; FERREIRA, M. A. Métodos para avaliação do grau de oxidação lipídica e da capacidade antioxidante. Química Nova, v. 22, n. 1, p. 94-103, 1999

37 SILVA, L.; PINTO, J.; CARROLA, J.; PAIVA-MARTINS, F. Oxidative stability of olive oil after food processing and comparison with other vegetable oils. Food Chemistry, v. 121, n. 4, p. 1177-1187, 2010.

38 SILVA, M. D.; OlIVEIRA, M. G. A.; LANNA, A. C.; PIRES, C. V.; PIOVESAN, N. D.; JOSÉ, I. C.; BATISTA, R. B.; BARROS, E. G.; MOREIRA, M. A. Caracterização da via das lipoxigenases em plantas de soja resistentes e susceptíveis a diaphorte phaseolorum f.sp. Meridionalis, agente causal do cancro-da-haste. Revista Brasileira de Fisiologia Vegetal, v. 13, n. 3, p. 316-329, 2001.

39 SILVA, R. F.; ASCHERI, J. L. R.; SOUZA, J. M. L. Influência do processo de beneficiamento na qualidade de amêndoas de castanha-do-Brasil. Ciência e Agrotecnologia, v. 34, n. 2, p. 445-450, 2010.

40 SOARES, D. J.; RODRIGUES, C. S.; MAIA, G. A.; SOUSA, P. H. M.; FIGUEIREDO, R. W. Avaliação sensorial de amêndoas de castanha de caju obtidas dos cultivos convencional e orgânico. Revista Brasileira de Produtos Agroindustriais, v.14, n.3, p. 245-250, 2012a.

41 SOARES, D. J.; CAVAlCANTE, C. E. B.; CARDOSO, T. G.; FIGUEIREDO, E. A. T.; MAIA, G. A.; SOUSA, P. H. M.; FIGUEIREDO, R. W. Study of the stability of cashew nuts obtained from conventional and organic cultivation. Semina: Ciências Agrárias, v.33, n.5, p. 1855-1868, 2012b.

42 SOTO-RODRÍGUEZ, I.; CAMPILLO-VELÁZQUEZ, P. J.; ORTEGA-MARTÍNEZ, J.; RODRÍGUES-ESTRADA, M. T.; LERCKER, G.; GARCIA, H. S. Cholesterol oxidation in traditional Mexican dried and deep-fried food products. Journal of Food Composition and Analysis, v. 21, n. 6, p. 489-495, 2008.

43 SOUZA JR., T. P.; OLIVEIRA, P. R.; PEREIRA, B. Exercício físico e estresse oxidativo. Efeitos do exercício físico intenso sobre a quimioluminescência urinária e malondialdeído plasmático. Revista Brasileira de Medicina do Esporte, v. 11, n. 1, p. 91-96, 2005.

44 TABEE, E.; AZADMARD-DAMIRCHI, S.; JAGESTAD, M.; DUTTA, P. C. Lipids and phytosterol oxidation in commercial French fries commonly consumed in Sweden. Journal of Food Composition and Analysis, v. 21, n. 2, p. 169-177, 2008.

45 TIRITAN, M. G.; BEUX, S. Controle da qualidade do óleo de soja degomado. Synerg Scyent, v. 1, n. 1-4, p. 306-316, 2006.

46 TRINDADE, M. A.; THOMAZINE, M.; OLIVEIRA J. M. de; BALIEIRO, J. C. C. de; FAVARO-TRINDADE, C. S. Estabilidade oxidativa, microbiológica e sensorial de mortadela contendo óleo de soja, armazenada a $0^{\circ} \mathrm{C}$ durante 60 dias. Brazilian Journal of Food Technology, v. 13, n. 3, p. 165-173, 2010. 
47 WARAHO, T.; Mc CLEMENTS, D. J.; DECKER, E. A. Mechanisms of lipid oxidation in food dispersions. Trends in Food Science \& Technology, v. 22, n. 1, p. 3-13, 2011.

48 ZUNIN, P.; LEARDI, R.; BISIO, A.; BOGGIA, R.; ROMUSSI, G. Oxidative stability of virgin olive oil enriched with carnosic acid. Food Research International, v. 43, n. 5, p. 1511-1516, 2010. 